\title{
Consideration of Dual Anti-platelet Therapy Duration after Drug-eluting Stent Implantation in a Japanese Population: A Five-year Follow-up after Sirolimus-Eluting Stent Implantation
}

\author{
Jun-ichi Kotani ${ }^{1}$, Yuji Ikari $^{2}$, Eishou Kyo ${ }^{3}$, Masato Nakamura ${ }^{4}$, \\ Hiroyoshi Yokoi ${ }^{5}$ and on behalf of the J-PMS investigators
}

\begin{abstract}
Objective To investigate the risks and benefits of prolonged dual antiplatelet therapy (DAPT: thienopyridine plus aspirin) following placement of drug-eluting stents (DES). The optimal duration of DAPT is not well established.

Methods We analyzed a prospective registry of 2,050 patients with sirolimus-eluting stents during a 5-year follow-up. We divided 1,691 patients into two groups according to DAPT duration (DAPT $\leq 12$ months ( $\mathrm{n}=$ $749)$ and DAPT $>12$ months ( $\mathrm{n}=942)$ ) and compared the clinical outcomes using a landmark analysis.

Results The frequencies of major adverse cardiac events (MACE: $15.6 \%$ vs. $18.2 \%)$, death $(10.0 \%$ vs. $11.5 \%)$, myocardial infarction ( $2.3 \%$ vs. $2.1 \%)$, target lesion revascularization $(4.5 \%$ vs. $6.3 \%)$ and stent thrombosis $(0.8 \%$ vs. $0.8 \%)$ were similar between the two groups. However, the frequency of bleeding was higher in the DAPT $>12$ months group ( $1.1 \%$ vs. $2.6 \%, \mathrm{p}=0.030)$. The adjusted 12 -month landmark analysis showed no differences in the incidence of MACE (hazard ratio (HR) $0.892 ; 95 \%$ confidence interval (CI) $0.689-1.155 ; \mathrm{p}=0.385$ ) or a composite of target vessel revascularization, cardiac death and myocardial infarction (target vessel failure: HR $0.922 ; 95 \%$ CI $0.678-1.255$; $\mathrm{p}=0.606$ ). There were no differences in the frequency of stent thrombosis between the two groups during years 2 to 5 after stenting; however, with regard to bleeding, an increase in the frequency of hemorrhage events was observed after four years from the index procedures in the DAPT $>12$ months group.

Conclusion DAPT performed beyond 12 months is associated with increased an frequency of bleeding complications and does not prevent the incidence of MACE, including stent thrombosis, during five years of follow-up after sirolimus-eluting stent implantation. Conducting larger, randomized studies will therefore be needed to confirm this finding.
\end{abstract}

Key words: follow-up study, stents, hemorrhage

(Intern Med 52: 703-711, 2013)

(DOI: 10.2169/internalmedicine.52.8205)

\section{Introduction}

Although the efficacy of drug-eluting stents is widely known, long-term safety concerns remain, especially regarding late stent thrombosis (1-3). Earlier studies have clarified that early discontinuation of dual antiplatelet therapy (DAPT: clopidogrel/ticlopidine and aspirin) is a risk factor for stent thrombosis in patients with drug-eluting stents $(4,5)$. However, the optimal duration of DAPT following drug-eluting stent implantation has not yet been well established. Unplanned DAPT continuation may increase the

${ }^{1}$ Cardiovascular Division, National Cerebral and Cardiovascular Center, Japan, ${ }^{2}$ Department of Cardiology, Tokai University School of Medicine, Japan, ${ }^{3}$ Department of Cardiology, Kusatsu Heart Center, Japan, ${ }^{4}$ Division of Cardiovascular Medicine, Toho University Ohashi Medical Center, Japan and ${ }^{5}$ Department of Cardiovascular Medicine, Kokura Memorial Hospital, Japan Received for publication May 21, 2012; Accepted for publication September 23, 2012 Correspondence to Dr. Jun-ichi Kotani, Shamallv8@aol.com 


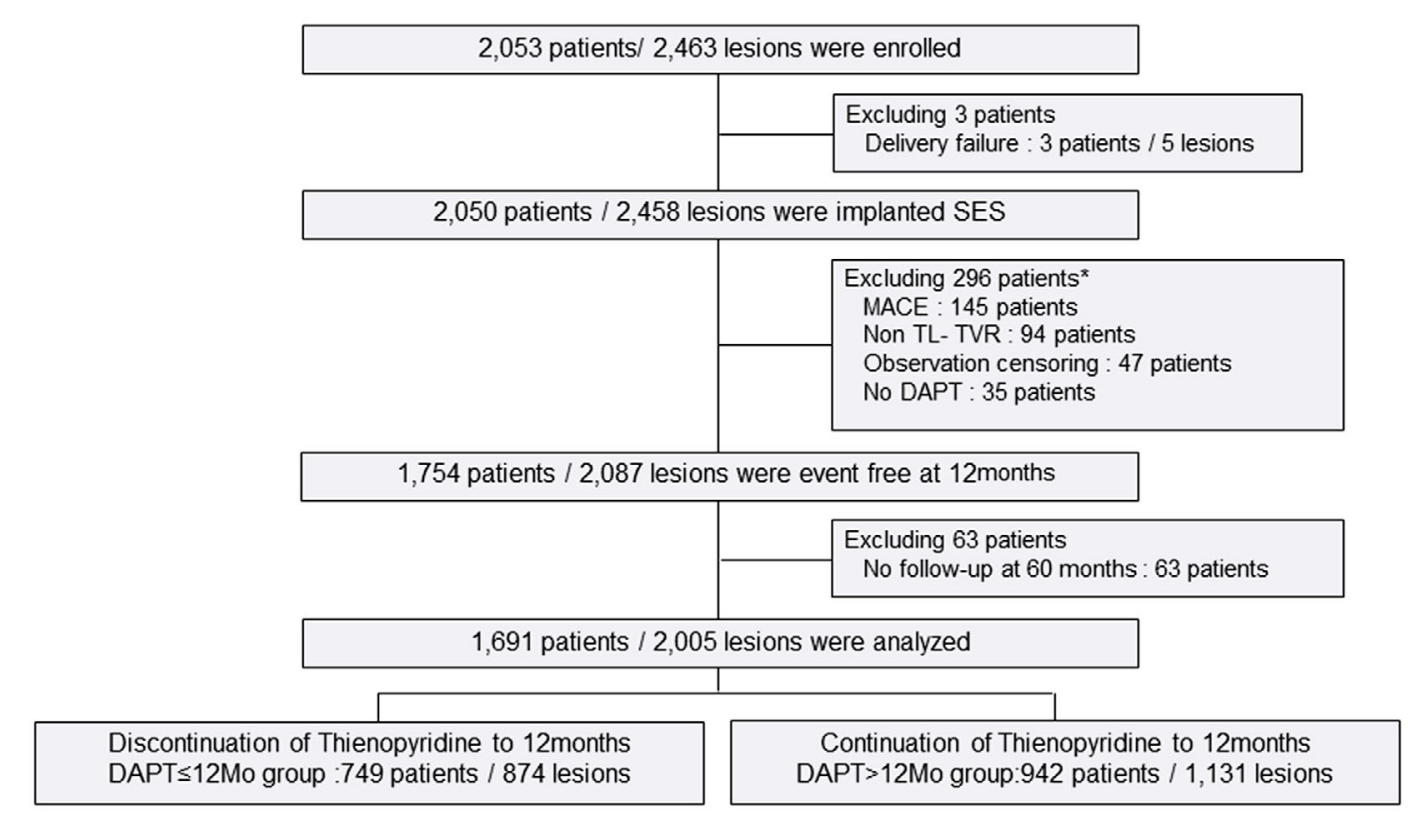

Figure 1. Schema of the 12-month landmark analysis. *: There were repetition patients. SES indicates sirolimus eluting stent, MACE: major adverse cardiac death, Non TL-TVR: non target lesiontarget vessel revascularization, DAPT: dual antiplatelet therapy

frequency of bleeding without decreasing the incidence of stent thrombosis. Since recent studies have clearly established that hemorrhagic complications from medications may offset the life-prolonging benefits of reduced ischemia, it is therefore important to carefully consider the risk/benefit ratio of prolonged DAPT. To address this issue, we compared differences in the frequencies of death, myocardial infarction (MI), late stent thrombosis and bleeding over five years after stent implantation between patients who received thienopyridine in addition to aspirin for up to one year and those treated for more than one year.

\section{Materials and Methods}

\section{Study population}

This analysis was conducted using the J-PMS database. JPMS is a prospective registry for post-marketing 5-year surveillance following index procedures whose creation was mandated by the Japanese government as one of the conditions for regulatory approval. The details of this program have been described previously (6). Briefly, the registry was planned to consecutively enroll a total of 2,053 patients who underwent sirolimus-eluting stent (SES) implantation (there were three delivery failures) between September 2004 and September 2005 at 50 community-based and university hospitals representative of the clinical environment in Japan. The indications for stent implantation were left to the discretion of each cardiologist participating in the registry and thus may have included off-label indications (acute MI, unprotected left main coronary arteries, bifurcation, ostial stenosis, restenotic lesions including in-stent restenosis, bypass grafts, chronic total occlusions, reference diameters less than $2.5 \mathrm{~mm}$, reference diameters greater than $3.5 \mathrm{~mm}$ or lesions greater than $30 \mathrm{~mm}$ in length).

\section{Data collection}

The patients' baseline characteristics, cardiac histories, risk factors, medications and procedural data were prospectively obtained and recorded by experienced research coordinators. Adjudication was based on the information contained in the case report forms and on the responses to queries addressed to the participating study sites. According to the study design, data were collected continuously for 30 days postprocedure at three, eight and 12 months, then annually from two to five years after the index procedures. The final follow-up ratio of this study was 1,937 out of 2,050 patients (94.5\%). Angiographic data for 1,063 of 2,458 lesions obtained at the time of the index procedures were analyzed quantitatively (QCA) by an independent core laboratory (Cardiocore, Tokyo, Japan). The study was approved by the institutional review board at each participating center. The information on DAPT discontinuation was collected via withdrawal reports, surveillance of prescription records or both. Termination of medications for at least 30 days constituted persistent discontinuation.

\section{Group assignments for landmark analysis}

A landmark analysis is a form of survival analysis that classifies patients based on some intermediate (nonoutcome) event occurring during follow-up. Prognosis is then evaluated from this landmark time point. We defined the landmark time and studied the outcomes in terms of the elapsed time from each patient's index procedure. In this study, the landmark time point was 12 months from the index procedure when thienopyridine was either continued or 
Table 1. Patient Characteristics

\begin{tabular}{|c|c|c|c|}
\hline & $\begin{array}{l}\text { DAPT } \leq 12 \mathrm{Mo} \\
\quad(\mathrm{n}=749)\end{array}$ & $\begin{array}{l}\text { DAPT }>12 \mathrm{Mo} \\
\qquad(\mathrm{n}=942)\end{array}$ & $\mathrm{p}$ \\
\hline Mean age, years & $67.0 \pm 9.9 \quad(749)$ & $67.2 \pm 9.8 \quad(942)$ & 0.643 \\
\hline Age $\geq 75$ years & $180 \quad(24.0)$ & $224 \quad(23.8)$ & 0.909 \\
\hline Male & $565 \quad(75.4)$ & $716 \quad(76.0)$ & 0.819 \\
\hline $\mathrm{LVEF}<30 \%{ }^{*}$ & $19 \quad(2.9)$ & $27 \quad(3.5)$ & 0.653 \\
\hline $\mathrm{BMI}, \mathrm{kg} / \mathrm{m}^{2}$ & $24.0 \pm 3.2 \quad(745)$ & $24.1 \pm 3.3 \quad(940)$ & 0.299 \\
\hline \multicolumn{4}{|l|}{ Type of disease } \\
\hline Stable ischemia & $488 \quad(65.2)$ & $636 \quad(67.5)$ & 0.325 \\
\hline AMI/UAP & $145 \quad(19.4)$ & $153 \quad(16.2)$ & 0.095 \\
\hline Previous MI & $269 \quad(35.9)$ & $364 \quad(38.6)$ & 0.266 \\
\hline Previous PCI & $376 \quad(50.2)$ & $550 \quad(58.4)$ & $<0.001 \dagger$ \\
\hline revious $\mathrm{CABG}$ & $44 \quad(5.9)$ & $86 \quad(9.1)$ & $0.013 \dagger$ \\
\hline Diabetes & $306 \quad(40.9)$ & $407 \quad(43.2)$ & 0.346 \\
\hline Treated with insulin & $65 \quad(8.7)$ & $94 \quad(10.0)$ & 0.402 \\
\hline Dialysis & $17 \quad(2.3)$ & $49 \quad(5.2)$ & $0.002 \dagger$ \\
\hline Hypertension & $504 \quad(67.3)$ & $674 \quad(71.6)$ & 0.062 \\
\hline Dyslipidemia & $446 \quad(59.6)$ & $540 \quad(57.3)$ & 0.372 \\
\hline Peripheral vascular disease & $38 \quad(5.1)$ & $69 \quad(7.3)$ & 0.070 \\
\hline Cerebrovascular disease & $51 \quad(6.8)$ & $73 \quad(7.8)$ & 0.511 \\
\hline Family history of CAD & $56 \quad(7.5)$ & $63 \quad(6.7)$ & 0.566 \\
\hline Current smoker & $142 \quad(19.0)$ & $181 \quad(19.2)$ & 0.901 \\
\hline Multi-vessel disease & $268 \quad(35.8)$ & $412 \quad(43.7)$ & $0.001 \dagger$ \\
\hline \multicolumn{4}{|c|}{$\begin{array}{l}\text { DAPT indicates dual antiplatelet therapy, LVEF: left ventricular ejection fraction, BMI: body mass index, AMI: acute } \\
\text { myocardial infarction, UAP: unstable angina pectoris, MI: myocardial infarction, PCI: percutaneous coronary } \\
\text { intervention, CABG: coronary artery bypass graft, CAD: coronary artery disease }\end{array}$} \\
\hline \multicolumn{4}{|c|}{$\begin{array}{l}\text { Values are expressed as mean } \pm \mathrm{SD}(\mathrm{n}) \text { or } \mathrm{n}(\%) \text {. The Wilcoxon rank sum test, Chi-square test or Fisher's exact test } \\
\text { were used as appropriate. }\end{array}$} \\
\hline$\dagger: \mathrm{p}<0.05$ were considered to indic & tical significance. & & \\
\hline
\end{tabular}

discontinued. Patients who remained event-free (no death, MI or revascularization) at 12 months and completed their first year of follow-up data collection, including questions regarding DAPT, were divided into two groups: thienopyridine discontinuation at or before 12 months and thienopyridine continuation (DAPT) beyond one year (Figure 1). The outcomes for these groups were evaluated up to five years (60 months) after initial percutaneous coronary intervention (PCI). There were 874 lesions in 749 patients who discontinued thienopyridine at 12 months or less (DAPT $\leq 12$ months) and 1,131 lesions in 942 patients who continued DAPT beyond 12 months (DAPT >12 months).

\section{Definition of events}

An independent safety and efficacy evaluation committee adjudicated all reported and suspected major adverse cardiac events (MACE) defined as: all-cause death, MI or target lesion revascularization (TLR) by PCI or coronary artery bypass graft surgery (CABG); stent thromboses; and target vessel revascularization (TVR) by PCI or CABG. Deaths were classified as cardiac or noncardiac based on SIRIUS trial protocol definitions; unidentified deaths, i.e., any death in which a cardiac cause could not be excluded, were classified as cardiac in this study. MI was classified as Q wave or non-Q wave according to electrocardiogram (ECG) changes and/or a rise in creatine kinase enzyme concentrations above twice the normal upper limit. If information for classifying the type of MI was not available, the MI was classified as unidentified. TLR was defined based on the SIRIUS trial protocol definition; all reported re-interventions inside an implanted stent or within $5 \mathrm{~mm}$ proximal or distal to a stent were classified as TLR. Repeated PCI in the same vessel but not TLR was recorded as non-target lesion revascularization (non-TL TVR). Target vessel failure (TVF) was defined as the combination of cardiac death, MI and TVR; therefore, this was considered a target vessel-related adverse event. Stent thrombosis was defined according to the Academic Research Consortium (ARC) definition, and both definite and probable cases were classified as stent thrombosis in this study. Because the current study was designed as a landmark analysis at 365 days, stent thrombosis indicated very late stent thrombosis (VLST) (7). Bleeding was defined according to the BARC (Bleeding Academic Research Consortium) definition, and Types 2, 3 and 5 were including in this study (8).

\section{Statistical analysis}

Continuous variables are expressed as the mean \pm standard deviation and categorical data are presented as frequencies. For comparisons between groups, the Pearson's Chi- 
Table 2. Lesion Characteristics and QCA Analysis

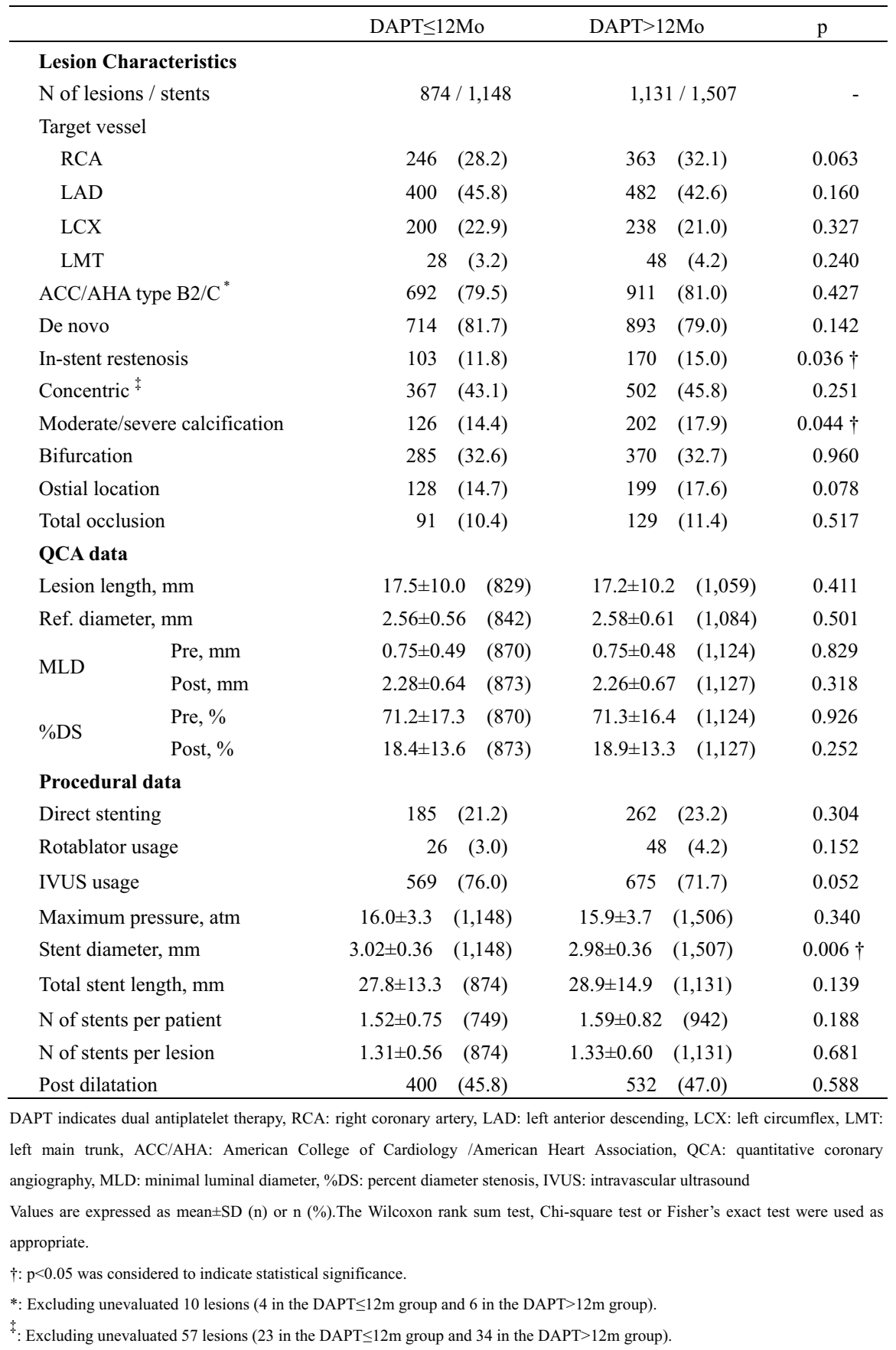

square test, Fisher's Chi-exact test or the Wilcoxon ranksum test were used as appropriate. Time-to-event data are presented using Kaplan-Meier estimates. Each event rate during the follow-up period was analyzed using the KaplanMeier method. A log-rank test was used for survival rate comparisons. The hazard ratios (HR) with 95 percent confidence intervals $(95 \% \mathrm{CI})$ were estimated from a Cox proportional hazards model. McNemar's test was used to determine whether there was a statistically significant difference between stent thrombosis and bleeding. Propensity score adjusting was used in the logistic regression models, with DAPT continuation at 12 months used as the dependent variable along with 27 independent variables (listed in the Supplemental tables). Patients with missing values for any given variable were excluded from the multivariable analysis. The goodness-of-fit of the propensity scores were assessed with the $\mathrm{C}$ statistic $(\mathrm{C}=0.605)$ and HosmerLemeshow tests $(\mathrm{p}=0.609)$. We calculated the adjusted hazard curves of the groups used in the landmark analysis using a Cox proportional hazards model in conjunction with previously described methods (9), adjusting for the propensity scores as a covariance. To additionally assess the adequacy of the propensity score adjustment, a sensitivity analysis using 1-to-many matching based on the propensity score of the final model was also performed and showed results similar to the primary analysis (data not shown). $\mathrm{P}$ values were two-sided, with $\mathrm{p}<0.05$ considered statistically significant. The statistical analyses were performed with SAS software, 


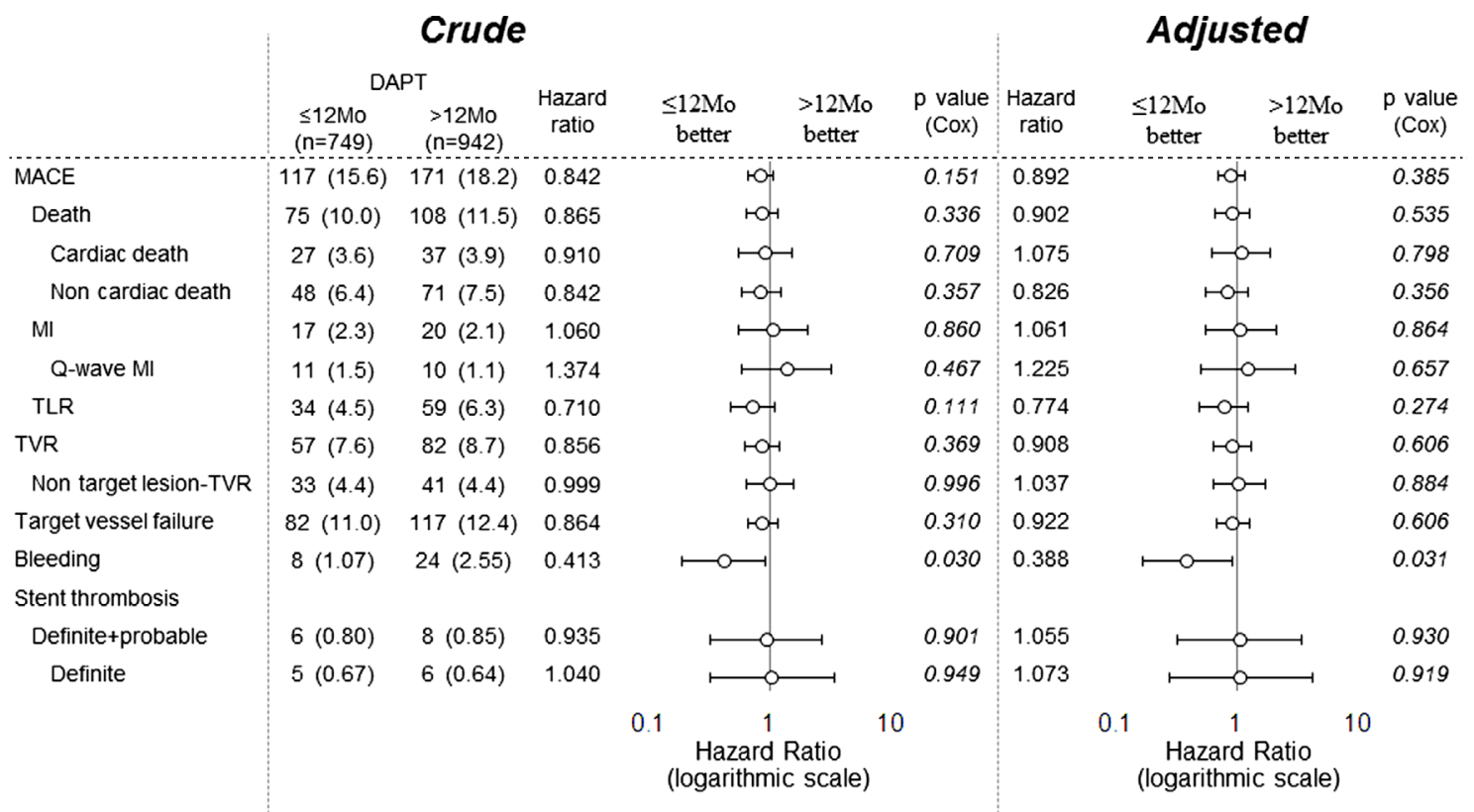

Figure 2. Crude and propensity score adjusting clinical results at 5-year. MACE indicates major adverse cardiac event, MI: myocardial infarction, CABG: coronary artery bypass graft, TLR: target lesion revascularization, TVR: target vessel revascularization
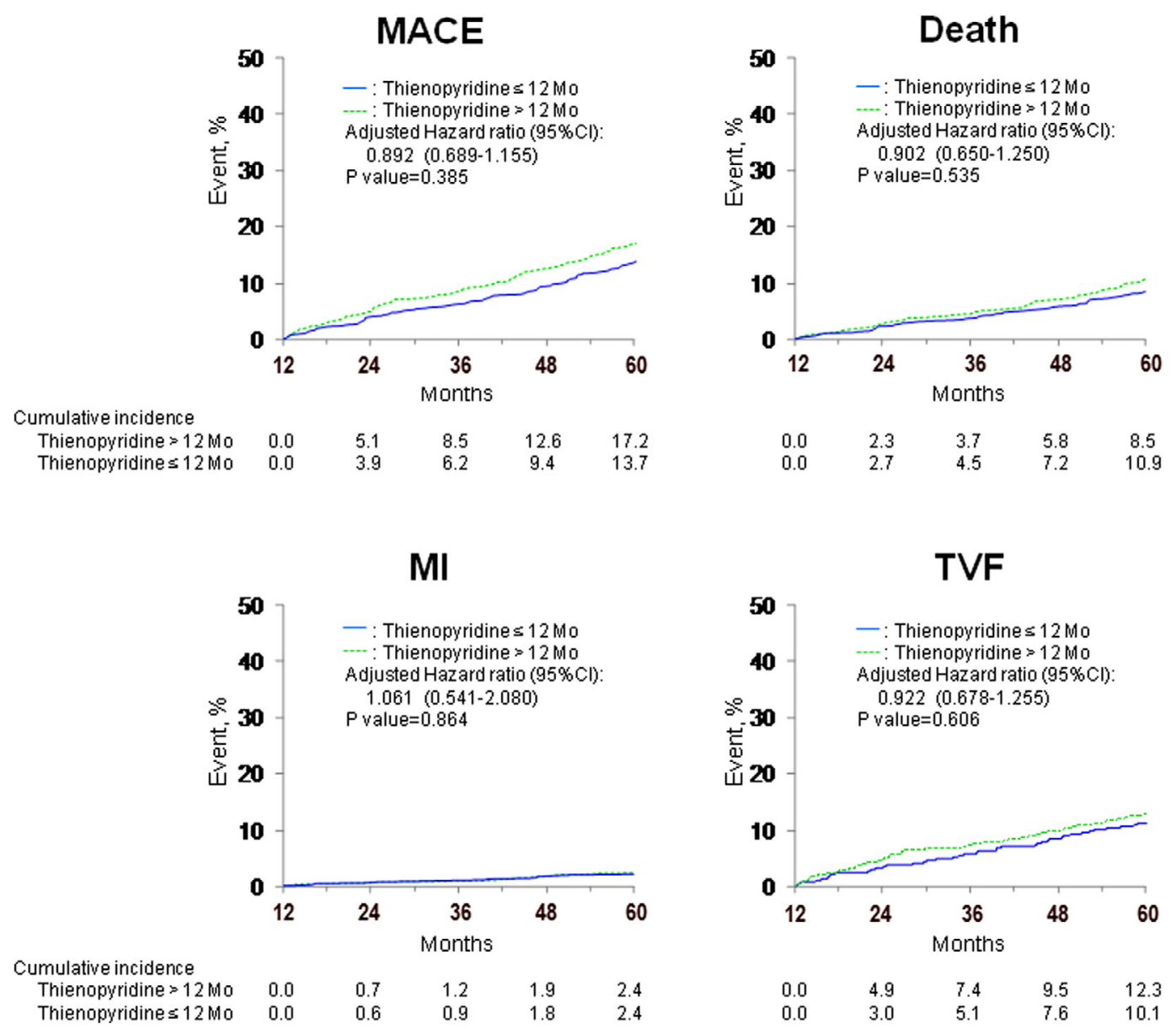

Figure 3. Adjusted cumulative incidences of major adverse cardiac event (MACE), death, myocardial infarction (MI) and target vessel failure (TVF) using the 12-months landmark analysis. 


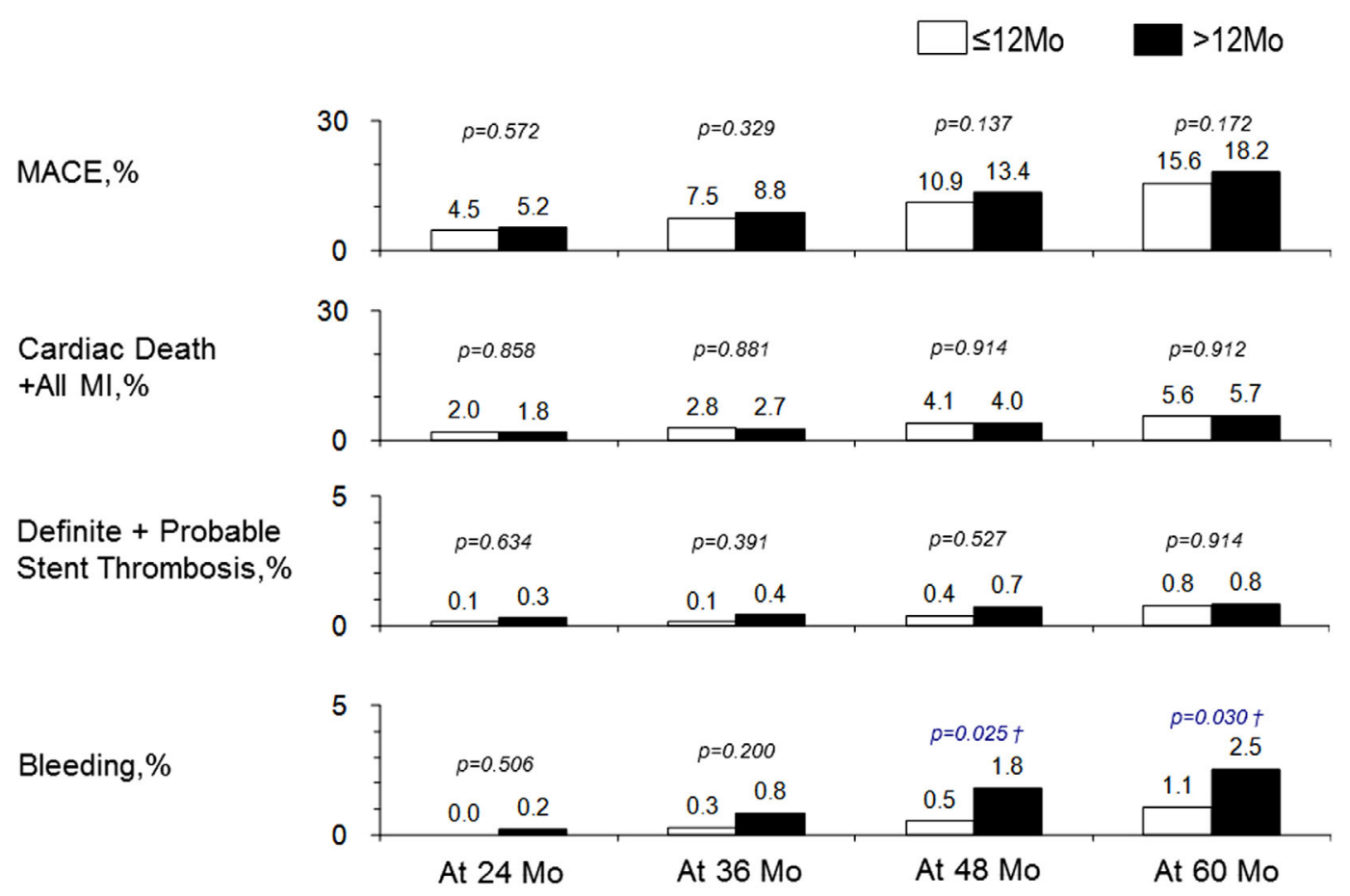

Figure 4. DAPT duration and incidence of risk (bleeding) and benefit (MACE, death, MI, stent thrombosis). DAPT indicate dual anti-platelet therapy, MACE: major adverse cardiac event, MI: myocardial infarction

Table 3. Incidence of Very Late Stent Thrombosis (VLST) and Bleeding Events

\begin{tabular}{lccccc}
\hline & $\begin{array}{c}\text { DAPT } \leq 12 \mathrm{Mo} \\
(\mathrm{n}=749)\end{array}$ & $\begin{array}{c}\text { DAPT }>12 \mathrm{Mo} \\
(\mathrm{n}=942)\end{array}$ & $\mathrm{p}$ \\
\hline VLST (Definite + probable) & 6 & $(0.80)$ & 8 & $(0.85)$ & 0.913 \\
$\quad$ & 5 & $(0.67)$ & 6 & $(0.64)$ & 0.938 \\
$\quad$ Definite & 1 & $(0.13)$ & 2 & $(0.21)$ & 0.698 \\
Probable & 8 & $(1.07)$ & 24 & $(2.55)$ & $0.030 \dagger$ \\
\hline
\end{tabular}

VLST indicates very late stent thrombosis.

Values are expressed as $n$ (\%). The Chi-square test or Fisher's exact test was used as appropriate. $\dagger: \mathrm{p}<0.05$ was considered to indicate statistical significance.

version 9.1 (SAS Institute Inc., Cary, NC).

\section{Results}

\section{Patient and lesion characteristics}

The patient characteristics are summarized in Table 1. There were no differences in patient characteristics, except that the DAPT $>12$ months group had significantly higher frequencies of past coronary revascularization, multi-vessel disease and current treatment with hemodialysis. Regarding procedural and QCA-related parameters, the rates of in-stent restenosis, the presence of moderate/severe calcification and the stent diameters were different between the two groups ( $\mathrm{p}=0.036, \mathrm{p}=0.044$ and $\mathrm{p}=0.012$ ), while other parameters were similar (Table 2). The frequency of "off-label" use in patients in the DAPT $\leq 12$ months and DAPT $>12$ months groups was also similar (632/749 patients, $84.4 \%$; and 808/ 942 patients, $85.8 \%$; $\mathrm{p}=0.449)$.

\section{Compliance with thienopyridine treatment}

Compliance with thienopyridine treatment in the DAPT $>12$ months group was obviously $100 \%$ at the 1-year follow-up. However, a steady decrease in compliance was observed thereafter, down to $84.6 \%$ at two years, $77.2 \%$ at three years, $70.0 \%$ at four years and $65.8 \%$ at five years.

\section{Long-term clinical outcomes and DAPT duration}

Fig. 2 shows the impact of thienopyridine administration in addition to aspirin beyond one year postprocedure on clinical outcomes. There were no clinical benefits of DAPT lasting more than 12 months. Rather, there was a marked increase in the frequency of bleeding complications associated with prolonged DAPT administration beyond one year; the crude data were $1.07 \%$ for the DAPT $\leq 12$ month group and $2.55 \%$ for the DAPT $>12$ month group ( $\mathrm{p}=0.03$ ), and the adjusted data also showed marked differences between the two groups (HR 0.413, 95\% CI 0.185-0.918, p=0.030).

\section{Landmark analysis based on DAPT at one year}

There were no differences in the cumulative incidences of MACE, death, MI and TVF from years 2 to 5 between the two groups (Figure 3). Similar results were observed for VLST up to year 5 in both groups; however, during the observation period, the cumulative incidence of bleeding events increased in the group receiving DAPT for more than 12 months (Figure 4). 


\section{Details of VLST and bleeding}

A total of 14 patients $(0.83 \%)$ presented with definite/ probable stent thrombosis and 32 patients (1.89\%) experienced bleeding events between years 2 and 5 (Table 3 ). Three patients who suffered VLST $(21.4 \%)$ and 11 patients who suffered bleeding $(34.3 \%)$ died within one day of the events. Two definite cases of VLST were observed after both antiplatelet medications were discontinued. In the DAPT $>12$ months group, five of eight cases of VLST $(62.5 \%)$ occurred under complete DAPT treatment. Regarding bleeding events, 12 patients experienced cerebral hemorrhage, 16 patients experienced gastrointestinal bleeding and four patients experienced hemorrhage from other sources (the lungs, mediastinum, intra-ocular areas or urinary tract). Mortality due to bleeding was $0.40 \%$ (3/749) in the DAPT $\leq$ 12 months group and $0.85 \%(8 / 942)$ in the DAPT $>12$ months group. The frequencies of both VLST and bleeding increased annually. The yearly slopes of the linear portions of the cumulative incidence curves between years 2 to 5 were $0.18 \%$ and $0.20 \%$ for VLST and $0.27 \%$ and $0.72 \%$ for bleeding, respectively.

\section{Discussion}

The results observed during our long-term observation period with a high follow-up rate $(95 \%)$ suggest that DAPT administration prolonged beyond one year increases the risk of bleeding without increasing benefits such as prevention of MACE or reduction of the frequency of VLST. Previous studies that addressed this issue did not conduct adequate assessments in terms of the observation periods or details of bleeding. The benefits of prolonged DAPT were thought to include the prevention of VLST, a specific phenomenon related to drug-eluting stents. However, only a study that compares two cohorts under long-term observation can sufficiently address this issue. Our current study shows that bleeding is also associated with prolonged DAPT administration.

Current ACC/AHA/SCAI recommendations call for one year of thienopyridine use to attenuate the risk of stent thrombosis after coronary artery drug-eluting stent implantation in patients not at high risk for bleeding (10). However, chronic thienopyridine use is costly and associated with an approximate $1 \%$ annual risk of major bleeding (11). Interestingly, a previous study showed that the predictors of bleeding are similar to those of stent thrombosis, including low ejection fraction, acute MI at presentation and multi-vessel PCI (12). Therefore, it is very important to explore the risk/ benefit ratio of DAPT duration. The results of previous large scale registries suggest that prolonged DAPT administration reduces the frequency of death and MI following DES implantation. This observation is consistent with recent reports from the Duke database (9) and findings in a diabetic population (13) where clopidogrel was discontinued at either six or 12 months. These results have led to uncertainty about the minimal necessary duration of DAPT after implantation of drug-eluting stents. Regarding the Duke database (9), the study design and analysis methods used in that database are similar to those used in the current study. However, the results of the Duke database suggest that extended use of clopidogrel in patients with DES may be associated with a reduced risk of death or $\mathrm{MI}$.

On the other hand, several observational and randomized studies of patients with drug-eluting stents have shown that continuation of clopidogrel therapy beyond six or 12 months does not reduce the risks of stent thrombosis or late clinical events, suggesting that DAPT can be discontinued approximately six to 12 months after performing index procedures (14-21). Two Korean randomized studies also previously reported lack of benefits of either 12 or 24 months of clopidogrel therapy over six or 12 months of clopidogrel therapy, respectively. These discrepancies may be explained by the following differences between the studies. The first difference is that the Duke database involved paclitaxeleluting stents (PES), while our database analyzed only SES. Regarding DES, there are several comparable studies showing SES to be superior to PES in terms of significant reductions in the risks of re-intervention (22) and stent thrombosis (23). The risk of death is not significantly different between the two types of DES; however, there is a trend toward a higher risk of MI with PES, especially after the first year following the procedure (14). The second difference is that the Duke database included a shorter follow-up period and lacked any assessment of hemorrhage. Our main finding is that an imbalance between the risks and benefits of DAPT are observed beyond 12 months. As shown in the figures, marked differences appeared starting at three years.

Regarding the follow-up period, the median follow-up terms used in the two Korean randomized studies were only 19.2 months (19) and 12 months (20), which might not have been sufficient to illustrate any trends of increasing risk. The reports that focused on both efficacy and the incidence of bleeding complications revealed that longer DAPT administration is not significantly more effective than 6-month DAPT administration in reducing all cause death, MI or cerebral accidents. Another study also demonstrated a greater risk of hemorrhage associated with 24 months of clopidogrel therapy in addition to aspirin (21). Stone et al. examined the effects of prolonged thienopyridine administration using data from the prospective, double-blind, randomized trials of the TAXUS series (24) and concluded that thienopyridine use beyond one year after drug-eluting stent implantation may reduce stent thrombosis over the subsequent 12-month period, although the rates of death and MI at two and five years are not reduced by extended thienopyridine use after either drug-eluting or bare-metal stenting. Further evaluation of the efficacy versus safety of extended DAPT duration (12 vs. 30 months) is being conducted by the DAPT (Dual AntiPlatelet Therapy) study (25). The optimal duration of DAPT after DES should be decided after taking into full consideration the risks and benefits assessed 
in that study.

The mechanisms underlying VLST have been investigated using invasive imaging and from a pathology-based approach (26-30). Previous studies suggest that delayed healing and local inflammation, including hypersensitivity or early atherosclerotic changes, may be responsible for the development of VLST, and some studies suspect a correlation between morphological changes and clinical thrombosis (28-30). A direct correlation between these chronic responses and late stent thrombosis can be easily assumed.

However, this and other studies have not shown the fact that prolonged continuation of DAPT markedly reduces the risk of clinical thrombosis $(19,21,31)$.

\section{Limitations}

This report is retrospective. The constraints of this study, inherent to any registry, include the lack of randomization, the absence of a control group and a lower level of monitoring than that used in standard randomized clinical trials, especially regarding the details of bleeding events. A serial comparison of laboratory values, e.g., hematocrit and hemoglobin, was not performed, which might have led to an underestimation of the frequency of bleeding complications. Even with adjustment, a landmark analysis is observational in nature and still subject to residual confounding (32). Despite any major differences in the groups at baseline, there may be a number of factors that led to the continuation or discontinuation of DAPT at 12 months. A proportion of the patients who discontinued DAPT after 12 months may have developed other problems prompting the discontinuation of clopidogrel therapy. These factors are in many ways impossible to control in a non-randomized study. A sample size of approximately 2,000 subjects is not sufficient to detect small but clinically meaningful differences in stent thrombosis rates; this would require 8,000-10,000 subjects. Additionally, there was no discrimination between ticlopidine and clopidogrel since the registry was started before clopidogrel was approved in Japan. The DAPT continuation group included patients who switched from ticlopidine to clopidogrel. However, previous studies have established that there are no differences in clinical efficacy between these two drugs (6), In addition, the duration of DAPT depended on each participating institution's practices. Finally, this analysis did not assess the efficacy of the systemic anti-atherosclerotic effects of clopidogrel.

\section{Conclusion}

Dual antiplatelet therapy, with the addition of thienopyridine to aspirin, extended beyond a period of 12 months, was thus found to increase the incidence of bleeding complications while not exhibiting any marked preventive effects on cardiovascular events, including stent thrombosis, during a five-year follow-up after sirolimus-eluting stent implantation.

The authors state that they have no Conflict of Interest (COI).

\section{References}

1. Daemen J, Wenaweser P, Tsuchida K, et al. Early and late coronary stent thrombosis of sirolimus-eluting and paclitaxel-eluting stents in routine clinical practice: data from a large twoinstitutional cohort study. Lancet 369: 667-678, 2007.

2. Bavry AA, Kumbhani DJ, Helton TJ, Borek PP, Mood GR, Bhatt DL. Late thrombosis of drug-eluting stents: a metaanalysis of randomized clinical trials. Am J Med 119: 1056-1061, 2006.

3. Lagerqvist B, James SK, Stenestrand U, Lindback J, Nilsson T, Wallentin L. Long-term outcomes with drug-eluting stents versus bare-metal stents in Sweden. N Engl J Med 356: 1009-1019, 2007.

4. Iakovou I, Schmidt T, Bonizzoni E, et al. Incidence, predictors, and outcome of thrombosis after successful implantation of drugeluting stents. JAMA 293: 2126-2130, 2005.

5. Park DW, Park SW, Park KH, et al. Frequency of and risk factors for stent thrombosis after drug-eluting stent implantation during long-term follow-up. Am J Cardiol 98: 352-356, 2006.

6. Ikari Y, Kotani J, Kozuma K, Kyo E, Nakamura M, Yokoi H; for the J-PMS Study Group. Assessment of sirolimus-eluting coronary stent implantation with aspirin plus low dose ticlopidine administration: one year result from the Cypher Stent Japan PostMarketing Surveillance Registry (J-PMS). Circ J 73: 1038-1044, 2009.

7. Cutlip DE, Windecker S, Mehran R, et al. Academic Research Consortium. Clinical end points in coronary stent trials: a case for standardized definitions. Circulation 115: 2344-2351, 2007.

8. Mehran R, Rao SV, Bhatt DL, et al. Standardized bleeding definitions for cardiovascular clinical trials: a consensus report from the bleeding academic research consortium. Circulation 123: 27362747, 2011.

9. Eisenstein EL, Anstrom KJ, Kong DF, et al. Clopidogrel use and long-term clinical outcomes after drug-eluting stent implantation. JAMA 297: 159-168, 2007.

10. King SB IIIrd, Smith SC Jr, Hirshfeld JW Jr, et al; 2005 WRITING COMMITTEE MEMBERS. 2007 Focused Update of the ACC/AHA/SCAI 2005 Guideline Update for Percutaneous Coronary Intervention: a report of the American College of Cardiology/ American Heart Association Task Force on Practice Guidelines. Circulation 117: 261-295, 2008.

11. Steinhubl SR, Berger PB, Mann JT III, et al; CREDO Investigators. Early and sustained dual oral antiplatelet therapy following percutaneous coronary intervention: a randomized controlled trial. JAMA 288: 2411-2420, 2002.

12. Kim YH, Lee JY, Ahn JM, et al. Impact of bleeding on subsequent early and late mortality after drug-eluting stent implantation. J Am Coll Cardiol Intv 4: 423-431, 2011.

13. Brar SS, Kim J, Brar SK, et al. Long term outcomes by clopidogrel duration and stent type in a diabetic population with de novo coronary artery lesions. J Am Coll Cardiol 51: 2220-2227, 2008.

14. Airoldi F, Colombo A, Morici N, et al. Incidence and predictors of drug-eluting stent thrombosis during and after discontinuation of thienopyridine treatment. Circulation 116: 745-754, 2007.

15. Park DW, Yun SC, Lee SW, et al. Stent thrombosis, clinical events, and influence of prolonged clopidogrel use after placement of drug-eluting stent data from an observational cohort study of drug-eluting versus bare-metal stents. JACC Cardiovasc Interv 1: 494-503, 2008.

16. Harjai KJ, Shenoy C, Orshaw P, Boura J. Dual antiplatelet therapy for more than 12 months after percutaneous coronary intervention: insights from the Guthrie PCI Registry. Heart 95: 1579-1586, 2009.

17. Kimura T, Morimoto $\mathrm{T}$, Kozuma K, et al; RESTART Investigators. Comparisons of baseline demographics, clinical presentation, and long-term outcome among patients with early, late, and very late 
stent thrombosis of sirolimus-eluting stents: observations from the registry of stent thrombosis for review and reevaluation (RESTART). Circulation 122: 52-61, 2010.

18. Lasala JM, Cox DA, Dobies D, et al; ARRIVE 1 and ARRIVE 2 Participating Physicians. Drug-eluting stent thrombosis in routine clinical practice: two-year outcomes and predictors from the TAXUS ARRIVE registries. Circ Cardiovasc Intervent 2: 285-293, 2009.

19. Park SJ, Park DW, Kim YH, et al. Duration of dual antiplatelet therapy after implantation of drug-eluting stents. N Engl J Med 362: 1374-1382, 2010.

20. Gwon H-C, Hahn J-Y, Park KW, et al. Six-months versus 12 month dual anti-platelet therapy after implantation of drug-eluting stents: the efficacy of Xience/Promus versus Cypher to reduce late loss after stenting (EXCELLENT) randomized, multicenter study. Circulation 125: 505-513, 2012.

21. Valgimigli M, Campo G, Monti M, et al; Ferrari R for PRODIGY investigators. Short- versus long-erm duration of dual-antiplatelet therapy after coronary stenting: a randomized multicenter trial. Circulation 125: 2015-2026, 2012.

22. Kastrati A, Dibra A, Eberle S, et al. Sirolimus-eluting stents vs paclitaxel-eluting stents in patients with coronary artery disease: meta-analysis of randomized trials. JAMA 294: 819-825, 2005.

23. Schomig A, Dibra A, Windecker S, et al. A meta-analysis of 16 randomized trials of sirolimus-eluting stents versus paclitaxeleluting stents in patients with coronary artery disease. J Am Coll Cardiol 50: 1373-1380, 2007.

24. Stone GW, Ellis SG, Colombo A, et al. Effect of prolonged thienopyridine use after drug-eluting stent implantation (from the TAXUS landmark trials data). Am J Cardiol 102: 1017-1022, 2008.

25. Mauri L, Kereiakes DJ, Normand SL, et al. Rationale and design of the dual antiplatelet therapy study, a prospective, multicenter, randomized, double-blind trial to assess the effectiveness and safety of 12 versus 30 months of dual antiplatelet therapy in subjects undergoing percultaneous coronary intervention with either drug-eluting stent or bare metal stent placement for the treatment of coronary artery lesions. Am Heart J 160: 1035-1041.e1, 2010.

26. Awata M, Kotani J, Uematsu M, et al. Serial angioscopic evidence of incomplete neointimal coverage after sirolimus-eluting stent implantation: comparison with bare-metal stents. Circulation 116: 910-916, 2007.

27. Imai M, Kadota $\mathrm{K}$, Goto $\mathrm{T}$, et al. Incidence, risk factors and clinical sequelae of angiographic peri-stent contrast staining after sirolimus-eluting stent implantation. Circulation 123: 2382-2391, 2011.

28. Cook S, Wenaweser P, Togni M, et al. Incomplete stent apposition and very late stent thrombosis after drug-eluting stent implantation. Circulation 115: 2426-2434, 2007.

29. Cook S, Ladich E, Nakazawa G, et al. Correlation of intravascular ultrasound findings with histopathological analysis of thrombus aspirates in patients with very late drug-eluting stent thrombosis. Circulation 120: 391-399, 2009.

30. Nakazawa G, Vorpahl M, Finn AV, Narula J, Virmani R. One step forward and two steps back with drug-eluting-stents. J Am Coll Cardiol Img 2: 625-628, 2009.

31. Kimura T, Morimoto T, Nakagawa $Y$, et al; j-Cypher Registry Investigators. Antiplatelet therapy and stent thrombosis after sirolimus-eluting stent implantation. Circulation 119: 987-995, 2009.

32. Dafni U. Primer on statistical interpretation and methods. Landmark analysis at the 25-year landmark point. Circ Cardiovasc Qual Outcomes 4: 363-371, 2011.

(C) 2013 The Japanese Society of Internal Medicine http://www.naika.or.jp/imonline/index.html 